\title{
The Population of Red Giant Stars in Globular Clusters of the Fornax Dwarf Galaxy
}

\author{
UFFE GRÅE JØRGENSEN ${ }^{1}$ and RAUL JIMENEZ ${ }^{2}$
}

${ }^{1}$ Niels Bohr Institute, University Observatory, Copenhagen, Denmark

${ }^{2}$ Royal Observatory, Edinburgh, U.K.

Fornax dSph is the only one of the Milky Way dwarf galaxies known to contain star clusters of its own, and the variety of its 6 clusters is as large as the variety among the many globular clusters in our own Galaxy. We have obtained CCD photometry of clusters 1 and 3 in $V$ and $I$ and through two narrow-band filters of the Wing system, designed to distinguish between carbon and M-type giants. The clusters contain a large population of carbon stars, in strong contrast to the (near) absence of carbon stars in the Galactic globular clusters. The carbon stars in the Fornax clusters populate only the lower $30 \%$ of the luminosity interval spanned by the Fornax field carbon stars. All the cluster carbon stars are fainter than the theoretical first helium shell flash luminosity. The large number of carbon stars could be explained by assuming the Fornax dSph to be about 6 billion years younger than the Galaxy, but this would still not explain their low luminosity. The $V-I$ colors of the giant branch stars define a much broader range than the corresponding very narrow Galactic globular cluster giant branches. The broad morphology can only be simulated by assuming a large metallicity spread among the Fornax giants, and we estimate that the individual stars along the giant branches span a range of metallicities all the way from $[\mathrm{Fe} / \mathrm{H}]=-2.4$ to $[\mathrm{Fe} / \mathrm{H}]=-1.6$.

The definite existence of carbon stars on the AGB in both of the observed Fornax clusters is a challenge to the theoretical models of the third dredge-up episode and to galactic chemical evolution models. It raises the central question of why the carbon stars in Fornax form already well below the theoretical carbon star luminosity limit, and also why they are present in relatively large numbers in the Fornax clusters and not at all in the Galactic globular clusters of the same metallicities.

Further details of this project are described by Jørgensen \& Jimenez (1997, $A \& A, 317,54)$. 